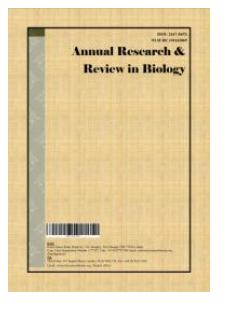

\title{
Parameterization and Forecasting of Childhood Pneumonia Model Using Least Square Approximation, Lagrange Polynomial and Monte Carlo Simulation
}

\author{
Cyrus Gitonga Ngari ${ }^{{ }^{*}}$ and Dominic Makaa Kitavi ${ }^{1}$ \\ ${ }^{1}$ School of Pure and Applied Sciences, Department of Mathematics, \\ Computing and Information Technology, University of Embu, P.O. Box, 6-60100, Embu, Kenya.
}

Authors' contributions

This work was carried out in collaboration between both authors. Author CGN designed the study, performed the statistical analysis, wrote the protocol and wrote the first draft of the manuscript. Author CGN managed the analyses of the study. Author DMK managed the literature searches. Both authors read and approved the final manuscript.

Article Information

DOI: $10.9734 / A R R B / 2020 / v 35 i 830265$ Editor(s):

(1) Dr. Gonzalo Emiliano Aranda Abreu, University of Veracruz, Mexico. Reviewers:

(1) Amruta Bhandari, R. C. Patel Institute of Technology, India. (2) Abdul Ghafoor Awan Dean, Institute of Southern Punjab, Pakistan. (3) Laxmi Narain Rohilla, J. C. Bose University of Science and Technology, YMCA, India and Echelon Institute of Technology, India. Complete Peer review History: http://www.sdiarticle4.com/review-history/58158

Original Research Article

Received 02 May 2020

Accepted 08 July 2020

Published 19 August 2020

ABSTRACT

Despite a study by [1] proposing a simple model of under five years pneumonia, doubt lingers regarding its reliability, sufficiency and validity. The research question is whether the model is valid for use or not? The objectives of this study were to: incorporate exit rate from under five-year age bracket in the model, use Kenya data to parameterize the model, taking into account the uncertainties and finally to predict the dynamics of pneumonia. The model was rescaled through nondimensionalization. Data was fitted using theory of general solutions of nonlinear Ordinary differential equations, numerical differentiation using Lagrange polynomials and least square approximation method. Uncertainties due to disparities and round off errors were simulated using Monte Carlo simulation. Predictions of dynamics of pneumonia were carried out using MATLAB inbuilt ode solvers. Excel software was used to predict dynamics of discrete ordinary differential 
equations and to fit data. The basic reproduction number $\left(R_{o}\right)$ and effective reproduction number $(R)$ were obtained as 61 and 7 respectively. Iteration of uncertainties on $R$ was carried out 1000 times by Monte Carlo simulation. The maximum and minimum $R$ were obtained as 90 and 55, respectively. Using MATLAB software and effective reproduction number, the ratio of infective class to the total population and the ratio of class under treatment to the total population will remain constant at 0.095 and 0.2297 respectively for the years 2021, 2022 and 2023. Research result indicted that it is more effective and efficient to use effective reproduction number $(R)$ than basic reproduction number $\left(R_{o}\right)$ in mathematical modelling of Infectious diseases whenever study focuses on proportion of population. On basis of large absolute errors in fitting data to model, findings cast doubt on model formulation and/or observed data.

Keywords: Childhood pneumonia; lagrange polynomial; parameterization; monte carlo; validation; forecasting.

\section{INTRODUCTION}

Pneumonia is infection of the lungs that is caused by fungi, bacteria, viruses, parasites or corrosive gas. It is characterized by inflammation of the alveoli in the lungs. Bacteria and viruses are the primary causes of pneumonia. Older adults, babies, and people with other diseases or impaired immune systems are more vulnerable to pneumonia [2].

Maternal HIV, male sex, maternal smoking, and malnutrition are associated with an increased incidence of pneumonia [3]. In Brazil, 1.5 million new cases of community-acquired pneumonia (CAP) occur in children every year, and the disease is ranked first on the list of hospitalization cases of these children in all regions of that country [4].

Childhood pneumonia is still a major public health issue for Kenya despite implementation of immunization in under five year's age bracket. It was still leading cause of death among underfive years, claiming equivalently $16 \%$ of child mortality in Kenya [5].

There are three popular techniques of carrying out numerical differentiation, these are: method based on interpolation, method based on undetermined coefficients and method based on finite difference operator. Lagrange polynomial $\left\{P_{n}(x)\right\}$ is one of techniques of method based on interpolations. Data with uniform or non-uniform mesh points, assumed to be $h(x)$ is first fitted in polynomials of the form $P_{n}(x)=$ $\sum_{k=0}^{n} \mathrm{~L}_{\mathrm{k}}(\mathrm{x}) \mathrm{f}_{\mathrm{k}}$ then $P_{n}^{\prime}(x) \cong h^{\prime}(\mathrm{x}) \quad[6,7]$. In the above, $P_{n}(x)$ denotes an $n$-degree polynomial in $x$.

Absolute mean and least square approximation are the most common method for approximating a linear and nonlinear function $(f)$ which may be known explicitly over given interval or be given in tabular form. The method is used to estimate parameters by minimizing the squared error obtained as difference between $(f)$ and the model $[6,8,9,6]$.

Monte Carlo method is based on generation of multiple trials to determine the expected value of a random variable which is uniformly distributed over the interval $[0,1]$. Beside a basic spread sheet program, Monte Carlo simulations can also be run by a number of commercial packages $[10,11]$. This study considered normally distributed random numbers.

Mathematical modelling is a process of representing essential features of reality using Mathematical language [12]. In most sciences, validation of hypothesis is done using experiments unlike in Mathematical epidemiology where experiments may be impossible and probably be unethical. However, formulating and analyzing biologically relevant disease models still poses a major challenge $[9,13]$

Mathematical modelling of infectious disease is a continuous ongoing process which is deemed to follow seven stages which are summarized in Fig. $1[13,9,12]$.

A model which undergoes all those stages up to analysis and forecasting leads to either a new generation of models or it is put in use. Generation of new models promises to keep mathematicians employed for considerable future time [13]. Though all the stages are vital, it should be emphasized that disease control models, which are to be used in control initiatives like Childhood pneumonia models, must have a realistic validation. This validation can only come from a comparison of model solutions and predictions with actual observed data [9]. 


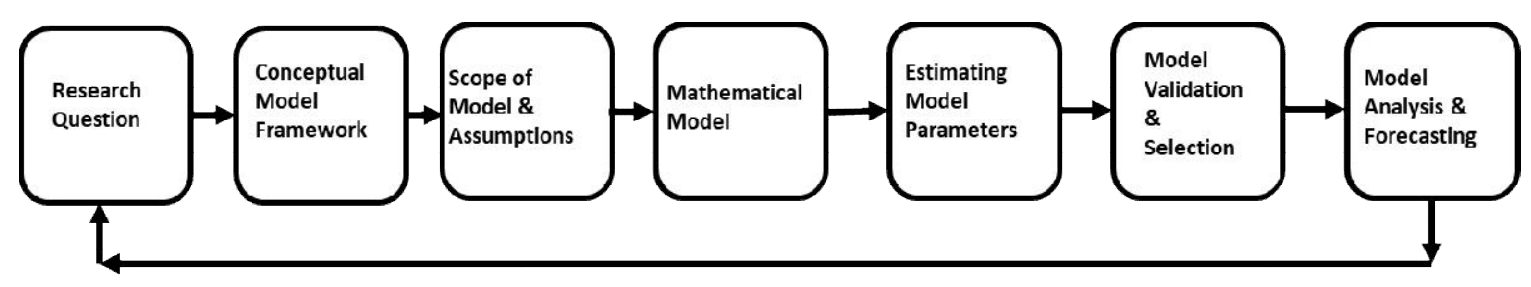

Fig. 1. Stages of modeling

A model by design should be as simple as possible but its simplicity should never compromise its initial objective of addressing a real-world problem. Though a simple model of childhood pneumonia that was proposed by [1] hypothesized a solution to perpetual problem of under five years pneumonia in Kenya and world at large. This developed model failed to: estimate parameters using real data, validate the model, forecast pneumonia dynamics, consider effective reproduction number and consider the rate of under five years children which exit that age bracket every year. This prompted this important question, "Is the model proposed by [1] fit for use? Or it should lead to a new generation of models?

The aim of this study was to: parameterize the model using Kenya data, validate or invalidate the model, carry out analysis, forecast; or propose generation of new pneumonia models. The paper is organized as follows. Section 2: Rescaling the model and model analysis, Section 3: Parameterization and Numerical analysis, Section 4: Discussion, and Section 5: Conclusion.

$$
\begin{aligned}
& \frac{d S}{d t}=\Pi-\beta\left(\frac{I+\eta T}{N}\right) \mathrm{S}-(\mu+\theta) \mathrm{S}+\gamma T+\tau I \\
& \frac{d I}{d t}=\beta\left(\frac{I+\eta T}{N}\right) \mathrm{S}-\Omega_{1} I \\
& \frac{d T}{d t}=\text { oI }-\Omega_{2} \mathrm{~T}
\end{aligned}
$$

\section{RESCALING THE MODEL AND MODEL ANALYSIS}

\subsection{Introduction}

According to [1], the model was formulated as follows, $P(t)$ was the total population of Kenya and $N(t)$ was the total population of under five years in Kenya which was divided into three subclasses: the susceptible class $S(t)$, the infective class $I(t)$ and class under treatment $\mathrm{T}(\mathrm{t})$. Other parameters were as follows: $\gamma$ was recovery rate, the recruitment rate of the Susceptible class was $\pi=$ birth rate $(b) \times N$, death due to disease occurred at a rate of $\alpha$ in infectious class, $\mu$ was the natural death rate, $\beta$ was the infection rates, $\partial_{1}$ and $\partial_{2}$ were death induced rates due to disease in Infected and treated classes respectively, o was the rate of treatment for children, $\mathrm{T}$ and $\mathrm{y}$ were the rate of recovery from infectious class and treated classes and $\theta$ is the exit rate from under five years age bracket. The force of infection per time was defined as $\lambda(\mathrm{t})=$ $\beta\left(\frac{I+\eta T}{N}\right)$. The dynamics of childhood pneumonia was described using first-order systems of equations as follows

Where,

$\Omega_{1}=\phi+\theta+\mu+\partial_{1}+\mathrm{T} ; \quad \Omega_{2}=\mu+\mathrm{\gamma}+\theta+\partial_{2}$;

$N(t)=S(t)+I(t)+\mathrm{T}(\mathrm{t}) ; \quad 0 \leq \eta \leq 1$.

\subsection{Rescaling the Model}

$$
\begin{aligned}
& \text { Let } t=t, s(t)=\frac{s(t)}{N(t)}, i(t)=\frac{I(t)}{N(t)} \text { and } r(t)=\frac{T(t)}{N(t)} \\
& \frac{d s}{d t}=\mathrm{b}-\beta(i+\eta r) \mathrm{s}-\mu \mathrm{s}+\gamma r+\tau i
\end{aligned}
$$




$$
\begin{aligned}
& \frac{d i}{d t}=\beta(i+\eta r) \mathrm{s}-\Omega_{1} i \\
& \frac{d r}{d t}=\text { qi }-\Omega_{2} \mathrm{r}
\end{aligned}
$$

Since,

$$
\begin{aligned}
& s=1-i-r \\
& \frac{\mathrm{di}}{\mathrm{dt}}=\beta(\mathrm{i}+\eta \mathrm{r})-\left\{\beta(\mathrm{i}+\eta \mathrm{r})(\mathrm{i}+\mathrm{r})+\Omega_{1} \mathrm{i}\right\} \\
& \frac{\mathrm{dr}}{\mathrm{dt}}=\phi \mathrm{i}-\Omega_{2} \mathrm{r}
\end{aligned}
$$

\subsection{Positivity and Boundness of the Solutions}

\subsubsection{Theorem 1.}

The region $\mathrm{R}$ given by $\mathrm{R}=\left\{[\mathrm{i}(\mathrm{t}), \mathrm{r}(\mathrm{t})] \in \mathrm{R}_{+}^{2} \mid \mathrm{s}(0) \geq\right.$ $0, \mathrm{i}(0) \geq 0, \mathrm{~b}=\mu\}$ is positively invariant and attracting with respect to the system of equations (7) - (8).

\subsubsection{Proof}

Let $(i, r)$ be any solution of the system with nonnegative initial conditions.

Consider, $\quad \frac{\mathrm{di}}{\mathrm{dt}}=\beta(\mathrm{i}+\eta \mathrm{r})-\{\beta(\mathrm{i}+\eta \mathrm{r})(\mathrm{i}+\mathrm{r})+$ $\Omega 1$ i. Clearly, $\beta i+\eta r \geq 0$, it follows that

$\frac{d i}{d t} \geq-\left\{\beta(i+\eta r)(i+r)+\Omega_{1} i\right\}$.

On

integration,

$i(t) \geq \mathrm{e}^{\int_{\mathrm{o}}^{\mathrm{t}}-\left\{\beta(\mathrm{i}(\mathrm{m})+\eta \mathrm{r}(\mathrm{m}))(\mathrm{i}(\mathrm{m})+\mathrm{r}(\mathrm{m}))+\Omega_{1} \mathrm{i}(\mathrm{m})\right\} \mathrm{dm}+c} \geq 0$, thus $\frac{\mathrm{di}}{\mathrm{dt}}$ stays positive.

Similarly, $\quad r(t)>r(0) \mathrm{e}^{-\Omega_{1} \mathrm{t}} \geq 0$. Therefore, system of equations (7) and (8) lie in the feasible region. The time derivative of our total population along its solution obtains: $\frac{\mathrm{ds}}{\mathrm{dt}}+\frac{\mathrm{di}}{\mathrm{dt}}+\frac{\mathrm{dr}}{\mathrm{dt}}=0=b-$ $\mu$. Therefore $b=\mu$.

\subsection{The Basic Reproduction Number $\left(R_{0}\right)$ and Effective Reproduction Number $\mathbf{R}$}

At disease-free equilibrium point (DFE), that is $E^{0}$ of the system (7) - (8), $i^{0}=r^{0}=0$.

This study used the Next-generation matrix method determines the basic reproduction number [14]. Let $f$ be a matrix of new infections terms and $v$ be the matrix of the remaining transfer of infections,

$f=\left(\begin{array}{c}\beta(\mathrm{i}+\eta \mathrm{r})-\beta(\mathrm{i}+\eta \mathrm{r})(\mathrm{i}+\mathrm{r}) \\ 0\end{array}\right), \mathrm{v}=\left(\begin{array}{c}\Omega_{1} i \\ -\phi p i+\Omega_{2} r\end{array}\right)$.

Matrices $\mathrm{F}$ and $\mathrm{V}$ are obtained by finding the Jacobian matrices of $f$ and $v$ and then evaluated at DFE point. The basic reproduction number is given by the spectral radius $\zeta$ (the dominant eigenvalue) of the matrix $\mathrm{FV}^{-1} \mathrm{FV}^{-1}$, denoted by $\zeta\left(\mathrm{FV}^{-1}\right)$ was obtain as $R_{0}=\beta\left(\frac{1}{\Omega_{1}}+\frac{\eta \phi}{\Omega_{1} \Omega_{2}}\right)$. The effective reproductive number $R=R_{0} \frac{S(t)}{P(t)}$.

\subsection{Existence of Endemic Equilibrium Point $\left(E^{*}\right)$}

\subsubsection{Theorem 2}

Endemic equilibrium point $\left(E^{*}\right)$ exist whenever $R_{0}>1$,

\subsubsection{Proof}

The endemic equilibrium point $E^{*}=\left\{\mathrm{i}^{*}, \mathrm{r}^{*}\right\}$ is determined by equating equations 7 and 8 to zero and solving for $\mathrm{i}^{*}$ and $\mathrm{r}^{*}$ in terms of $\mathrm{R}_{0}$ and $\mathrm{R}$. They are obtained as,

$$
\begin{aligned}
& \mathrm{i}^{*}=\frac{\Omega_{2}\left(-\eta \phi+\phi \eta R_{0}+\left(-1+R_{0}\right) \Omega_{2}\right)}{R_{0}\left(\phi+\Omega_{2}\right)\left(\phi p+\Omega_{2}\right)} \text { or } \mathrm{i}^{*}=\frac{\Omega_{2}\left(-\eta \phi+\phi \eta R+(-1+R) \Omega_{2}\right)}{R\left(\phi+\Omega_{2}\right)\left(\phi p \eta+\Omega_{2}\right)} \text { and } \\
& \mathrm{r}^{*}=\frac{\phi\left(-\eta \phi+\phi \eta R_{0}+\left(-1+R_{0}\right) \Omega_{2}\right)}{R_{0}\left(\phi+\Omega_{2}\right)\left(\phi \eta+\Omega_{2}\right)} \text { or } \mathrm{r}^{*}=\frac{\phi\left(-\eta \phi+\phi \eta R+(-1+R) \Omega_{2}\right)}{R\left(\phi+\Omega_{2}\right)\left(\phi \eta+\Omega_{2}\right)} . \text { Also, at } E^{*},
\end{aligned}
$$




$$
\lambda^{*}=\frac{\Omega_{1} \Omega_{2}\left(-\eta \phi p+\phi p \eta R_{0}+\left(-1+R_{0}\right) \Omega_{2}\right)}{\left(\phi p+\Omega_{2}\right)\left(\eta \phi p+\Omega_{2}\right)} \text { or } \lambda^{*}=\frac{\Omega_{1} \Omega_{2}\left(-\eta \phi p+\phi p \eta R+(-1+R) \Omega_{2}\right)}{\left(\phi p+\Omega_{2}\right)\left(\eta \phi p+\Omega_{2}\right)}
$$

Endemic equilibrium exists whenever $\lambda^{*}>0$, that is whenever $R>1$ or $R_{0}>1$.

The condition necessary and sufficient for local and global stability were established [1].

\section{MODEL PARAMETERIZATION, VALIDATION AND COMPARISON}

\subsection{Parameterization}

The number of births for every 1000 people in Kenya from year 2010 to 2017 were:35.09, $34.34,33.61,32.93,32.32,31.78,31.31$ and 30.88 [2]. Note that $\pi=$ birth rate $(\mathrm{b}) \times$ Total population $(\mathrm{N})$. The birth rate was obtained as mean of birth rate from 2010 to 2017 therefore $b \cong 0.0328$ with standard error of 0.0015 .

Under five years is a period from 0 to 59.999 months, which is about 59.999/12 years. The exit rate from that age bracket per year is estimated as $\frac{12}{59.999}$ year $^{-1}$. Pneumonia had accounted for $15 \%, 16 \%$ and $16 \%$ of under-five deaths for years 2017, 2016 and 2014, whose mean is $15.67 \%$ and standard error of $0.58 \%$ [5]. The under-five mortality rate per 1000 from year 2010 to 2017 was: $58.4,56.3,54.4,52.3,50.51$, 48.7, 47.1 and 45.6, whose mean was 0.0517 and standard error of 0.0045 [5]. Therefore, Pneumonia accounted for $15.67 \%$ and other causes $84.33 \%$. Death due to other cause is approximated as $84.33 \%$ of $0.0517 \cong 0.0436$. Therefore $\mu \cong 0.0436$ The rate of pneumonia induced death for year 2012 and 2013 at inpatient classes was 0.0511 and 0.0526 respectively, mean 0.0519 and standard error of $0.0011[15]$.

Total Treated $r(t)=\frac{T_{n}(t)+T_{S}(t)}{N(t)}$

Estimate 1 (E1): Pneumonia prevalence was estimated at $16.1 \%$ of total under five population in Ethiopia which neighbors Kenya [18]. Then $\mathrm{S}(\mathrm{t})$ was estimated as: 83.9\% of $N(t)$ and $16.1 \%$ of $N(t)=I_{n}(t)+I_{s}(t)+$ $T_{n}(t)+T_{s}(t)$.

Estimate 2 (E2): Data for outpatients was not categorized as under five years but was provided for all ages as bloc as 253,731; 552,718; 535,$024 ; 576,703$ for years $2010,2011,2012$, and 2013 respectively. The mean proportion of under five years' inpatient to total outpatient cases for the same period 2010 to 2013 was obtained as 0.6343 with standard error as 0.0376 . This proportion was used to estimate under five years for outpatient. After getting estimate for under five years' outpatient, the mean proportion of inpatient to outpatient for period 2010 to 2013 was obtained as 0.0136 with standard error as 0.0100 . The proportion for 2010 to 2013 was used to estimate under five years' inpatient from 2014 to 2017 and also severely and non-severely infected under five years children, that is $I_{S}(t)=0.0136 I_{n}(t)$. Note that $I_{n}(t)+I_{S}(t)=$ $N(t)-T_{s}(t)-T_{n}(t)$

Equations (7) and (8) are expressed as

$$
\begin{aligned}
& \frac{\mathrm{d}}{\mathrm{dt}}\left(\ln \frac{i(t)}{i(0)}\right) \\
& =\beta\left(1+\eta\left[\frac{r(t)}{i(t)}-r(t)-\frac{r(t)^{2}}{i(t)}\right]-i(t)-r(t)\right)-\left(\phi+\frac{12}{59.999}+0.0436+\partial_{1}\right. \\
& +\mathrm{T}) \\
& \frac{\mathrm{d}}{\mathrm{dt}}\left(\ln \frac{r(t)}{r(0)}\right)=\phi \frac{i(t)}{r(t)}-\left(0.0436+\mathrm{v}+\frac{12}{59.999}+0.0519\right) \\
\text { Since } \theta= & \frac{12}{59.999} \text { year }^{-1} ; \mu=0.0436 ; \partial_{2}=0.0519 ;
\end{aligned}
$$

Let 2010 be $\mathrm{t}=0$ and 2017 be $\mathrm{t}=7$, then $\ln \frac{i(t)}{i(0)}$ is obtained by fitting values in the table in $7^{\text {th }}$ degree polynomial using MATLAB: 
Ngari and Kitavi; ARRB, 35(8): 102-114, 2020; Article no.ARRB. 58158

Table 1. Available population data and estimations of unknown values $\{E i\}$

\begin{tabular}{|c|c|c|c|c|c|c|c|c|}
\hline Year/Variable & 2010 & 2011 & 2012 & 2013 & 2014 & 2015 & 2016 & 2017 \\
\hline $\begin{array}{l}S(t) \times 10^{6} \\
\{\mathrm{E} 1\}\{83.9 \% N(t)\}\end{array}$ & 4.6145 & 3.3604 & 5.8360 & 5.9132 & 3.8406 & 4.7779 & 6.1263 & 7.8985 \\
\hline$I_{n}(t)\{\mathrm{E} 2\}$ & 714753 & 284022 & 762235 & 754460 & 111856 & 285934 & 545955 & 1045393 \\
\hline$I_{S}(t)\{\mathrm{E} 2\}$ & 9721 & 3863 & 10367 & 10261 & 1522 & 3889 & 7425 & 14218 \\
\hline $\mathrm{i}(\mathrm{t})$ & 0.131722545 & 0.071876014 & 0.111069868 & 0.108501844 & 0.024768001 & 0.050893462 & 0.075785754 & 0.112554545 \\
\hline$T_{n}(t)$ & $160940\{\mathrm{E} 2\}$ & $350590\{\mathrm{E} 2\}$ & $339370\{E 2\}$ & $36580\{\mathrm{E} 2\}$ & 615254 & 618564 & 613871 & 449969 \\
\hline$T_{s}(t)\{\mathrm{E} 2\}$ & 86 & 6425 & 8028 & 4279 & $8368\{\mathrm{E} 2\}$ & $8413\{\mathrm{E} 2\}$ & $8349\{\mathrm{E} 2\}$ & $6120\{\mathrm{E} 2\}$ \\
\hline$r(t)$ & 0.02927746 & 0.08913565 & 0.04994221 & 0.05250837 & 0.13623340 & 0.11009834 & 0.08521344 & 0.04844692 \\
\hline$N(t) \times 10^{6}$ & 5.5 [16] & 4.0053 & 6.956 [16] & $7.0480[16]$ & $4.5776\{\mathrm{E} 3\}$ & $5.6947\{\mathrm{E} 3\}$ & $7.3019\{\mathrm{E} 3\}$ & $9.4142\{\mathrm{E} 3\}$ \\
\hline $\begin{array}{l}\text { Kenya } \\
\text { Population }(\mathrm{P}(\mathrm{t})) \\
\times 10^{6}[17]\end{array}$ & 41.3502 & 42.4868 & 43.6466 & 44.8268 & 46.0243 & 47.2363 & 48.4616 & 49.6999 \\
\hline
\end{tabular}


$\ln \frac{i(t)}{i(0)}=-0.0032 t^{7}+0.075 t^{6}-0.68 t^{5}+3 t^{4}-6.1 t^{3}+5 t^{2}-0.064 t+1.2 \times 10^{-12} ; \quad$ Norm of residuals $=1.3694 \times 10^{-12}$. Therefore

$$
\frac{\mathrm{d}}{\mathrm{dt}}\left(\ln \frac{i(t)}{i(0)}\right) \cong-0.0224 t^{6}+0.45 t^{5}-3.4 t^{4}+12 t^{3}-18.3 t^{2}+10 t-0.064
$$

Similarly,

$$
\begin{gathered}
\begin{array}{c}
\ln \frac{r(t)}{r(0)}=0.0069 t^{7}-0.17 t^{6}+1.6 t^{5}-7.2 t^{4}+17 t^{3}-18 t^{2}+6.5 t-2.7 \\
\quad \times 10^{-12} ; \text { norm of residuals }=3.4628 \times 10^{-12}
\end{array} \\
\frac{\mathrm{d}}{\mathrm{dt}}\left(\ln \frac{r(t)}{r(0)}\right) \cong 0.0483 t^{6}-1.02 t^{5}+8 t^{4}-28.8 t^{3}+51 t^{2}-36 t+6.5
\end{gathered}
$$

Fig. 2. The data was fitted using Excel software least square solver:

a) Mean least square error of 1326.284 and standard deviation of least square error of 1873.4.

b) Mean absolute error of 27.7923208 and standard deviation of absolute error of 25.1594 . Parameters were estimated as $\beta=29 ; \eta=0.455 ; \phi=0.9 ; \partial_{1}=0.0004 ; \mathrm{T}=0.006$

Fig. 3. The data was fitted using Excel software solver:

a) Mean least square error of 3726.074 and standard deviation of least square error of 5546.334415

b) Mean absolute error of 43.19271 and standard deviation of absolute error of 46.11121 . Parameters were estimated as $\phi=0.9 ; \gamma=0.00001$

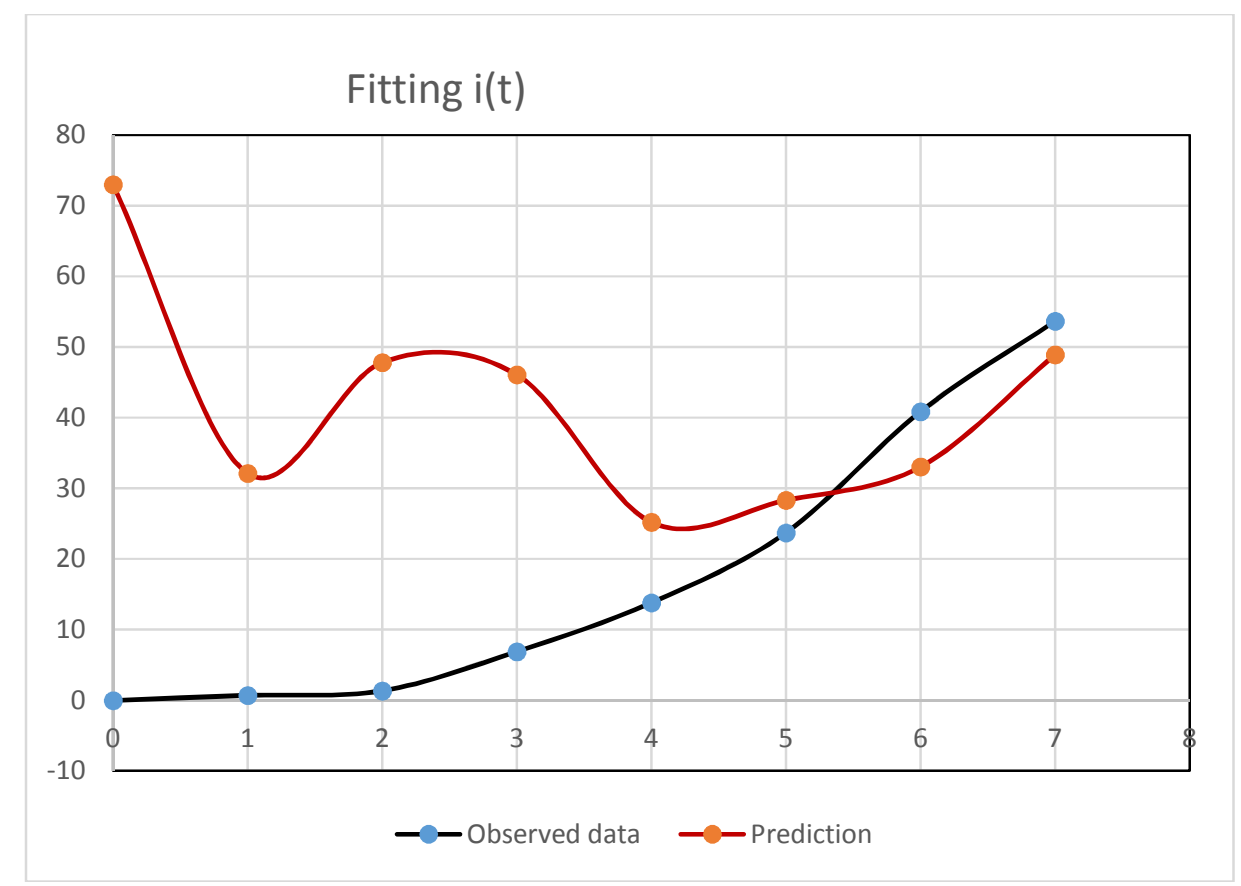

Fig. 2. Fitting $i(t)$ to observed data 


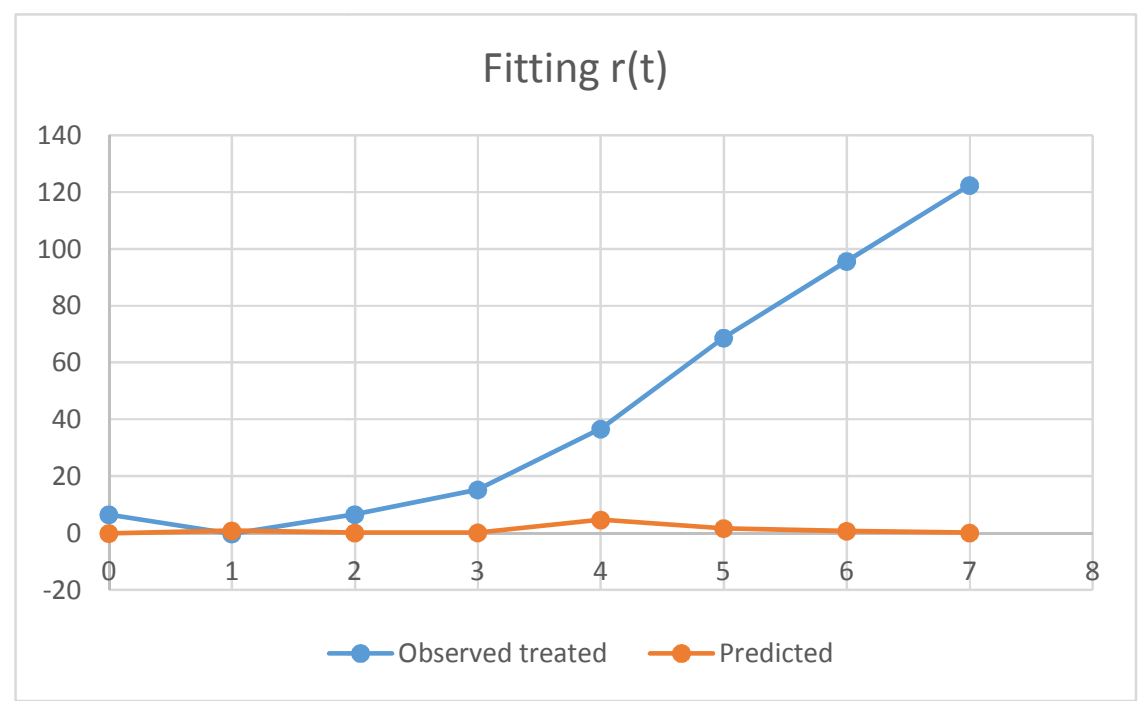

Fig. 2. Fitting $r(t)$ to observed data

\subsection{Simulations}

Simulations were carried out using inbuilt MATLAB ordinary differential equation solver. Average $\frac{S(t)}{P(t)}=0.116759$ with standard error as $\mp 0.029109$ using population data in Table 1.

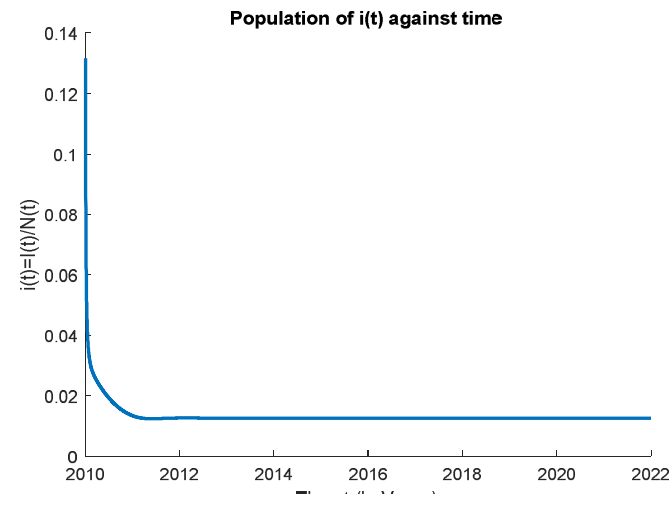

Fig. 3. Simulation of $i(t)$ using Ro

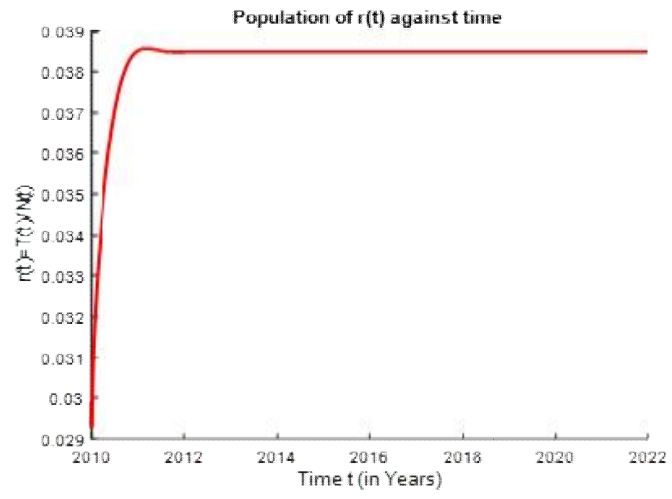

Fig. 5. Simulation of $r(t)$ using Ro

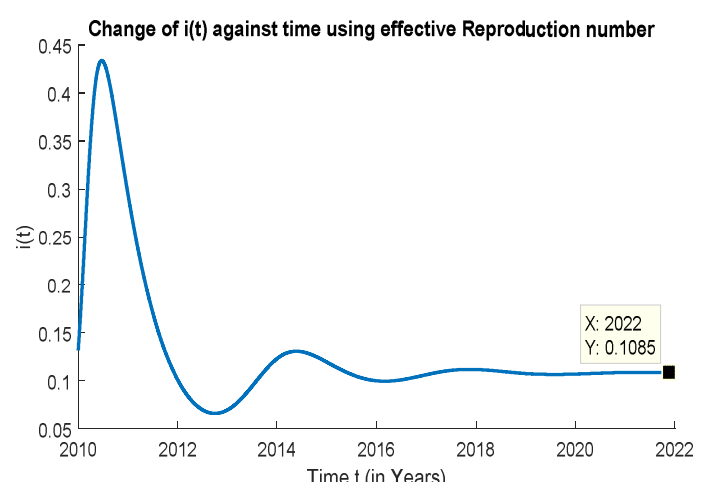

Fig. 4. Simulation of $i(t)$ using $\mathrm{R}$

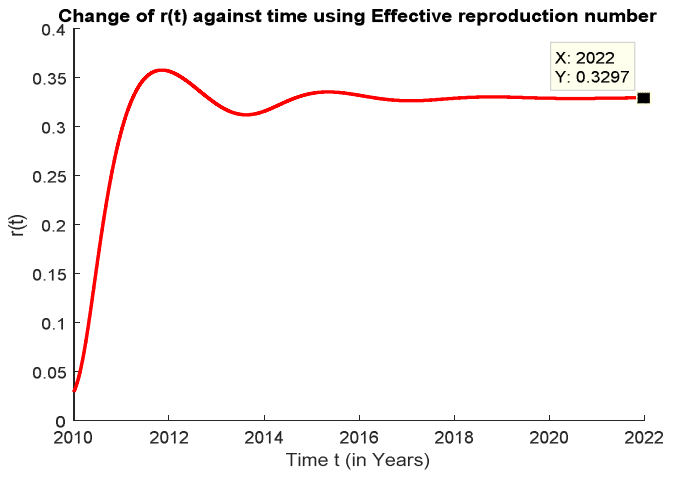

Fig. 6. Simulation of $r(t)$ using $R$ 
The basic reproduction number $\left(R_{0}\right)$ is estimated as 61 and effective Reproduction number (R) as $61 \times \frac{S(t)}{P(t)}$. Equations 7 and 8 can be expressed as discrete ordinary differential equations by incorporating $R_{0}$ and $R$ as below

$$
\begin{gathered}
i(t+1)=R_{0} \mathrm{i}(\mathrm{t})-\Omega_{1} \mathrm{i}(\mathrm{t}) \\
\mathrm{r}(\mathrm{t}+1)=\operatorname{di}(\mathrm{t})-\Omega_{2} \mathrm{r}(\mathrm{t}) \\
i(t+1)=R_{0} \frac{S(t)}{P(t)} \mathrm{i}(\mathrm{t})-\Omega_{1} \mathrm{i}(\mathrm{t})
\end{gathered}
$$

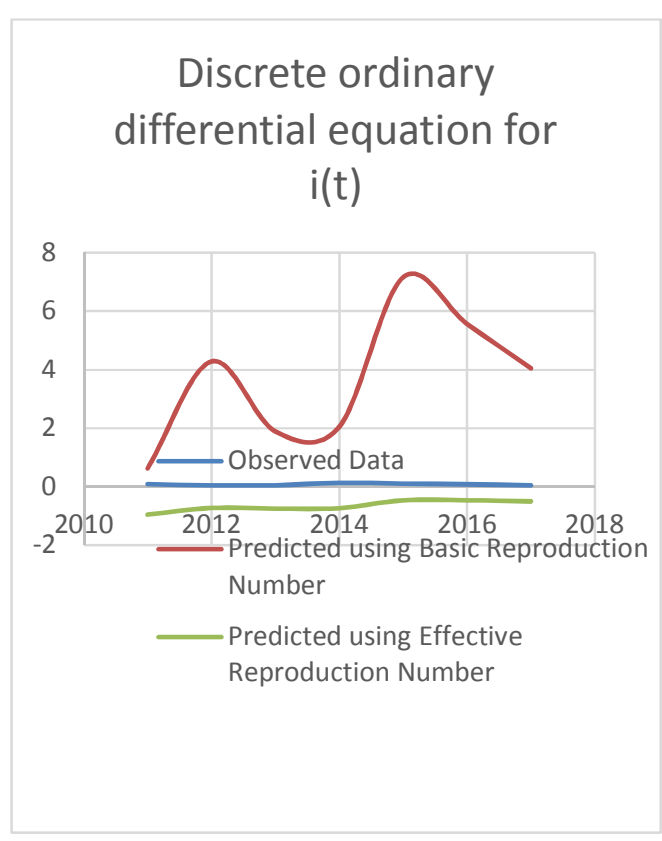

Fig. 7. Discrete $i(t)$ fitted using $\mathrm{R}$ and Ro

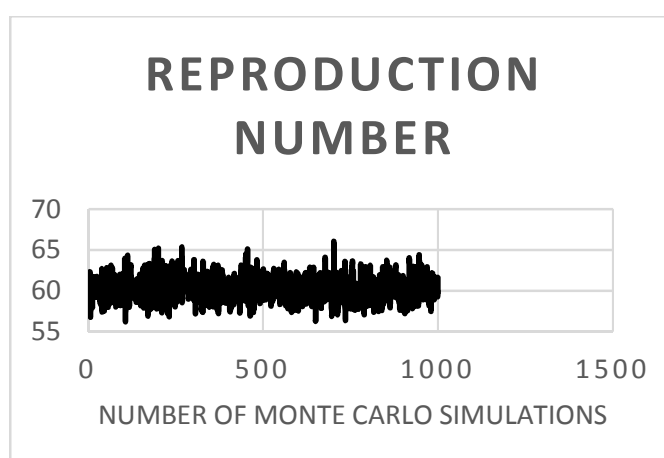

Fig. 9. Simulated $R$ with $S(t) / N(t)$ constant

The above discrete equations were fitted to the observed data using Excel least square Solver to obtain Figs. 8 and 9.

\section{Discrete Ordinary differential equations for $r(t)$}

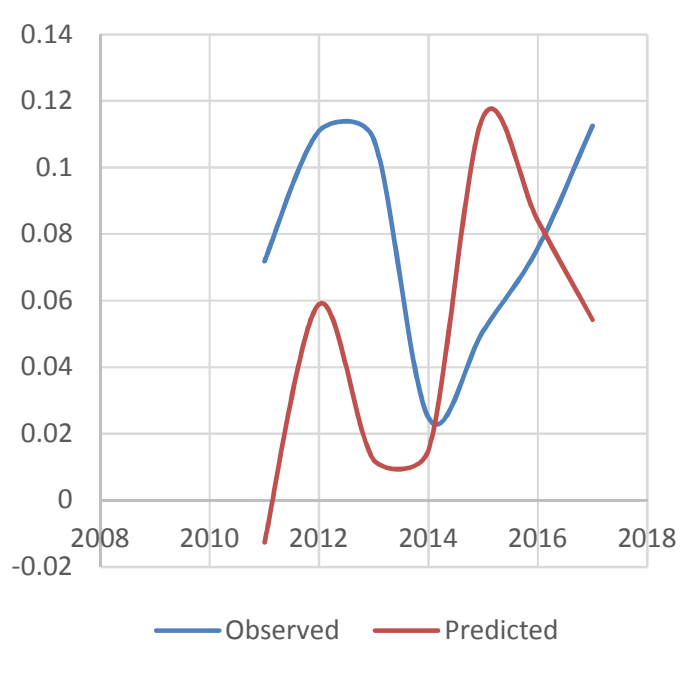

Fig. 8. Discrete $r(t)$ fitted using $R$ and Ro



Fig. 10. Simulated $R$ with $S(t) / N(t)$ uncertain

Fig. 8. Mean absolute error were 3.582191 and 0.730658 for $\mathrm{R} 0$ and $\mathrm{R}$ respectively,

Fig. 9. Mean absolute error was 0.05334 . 


\subsection{Monte Carlo Simulation using Normally Distributed Random Numbers}

All systems are prone to variability due to either assignable causes or chance causes, or both. So, for this model to be tractable mathematically, we shall assume variability in state variables of the rescaled model, that is $i(t)$ and $r(t)$. Suppose we investigate how random effects and round off errors would affect the basic reproduction number and effective reproduction number using Monte Carlo simulations. The study assumed normally distributed random numbers and the iterations were carried out using Excel software to obtain Figs. 10 and 11 .

\subsection{Bifurcation Analysis Using Numerical Method}

Numerical analysis of the system of equations 7 and 8 using the estimated parameters in the neighborhood of $R_{0}=1$ confirms that achieving the condition $R_{0}<1$ is sufficient to eradicate pneumonia and that the system does not exhibit backward bifurcation but a forward bifurcation [13].

From Figs. 13 and 14 obtained using Mathematica, $\quad i(t)<0$ and $\lambda(t)<0$ are not biologically meaningful and therefore should be construed to mean $i(t)=0$ and $\lambda(t)=0 s$ ince $i(t) \geq 0$ and $\lambda(t) \geq 0$.

\subsection{Normalized Sensitivity Analysis of Basic Reproduction Number}

Sensitivity analysis is ordinarily used to determine the vigor of model forecasts to parameter values, since there are usually errors in data collection methods and supposed parameter values. It is used to ascertain parameters that have a high effect on $R_{o}$ and should be targeted by control approaches. The normalized forward sensitivity index of $R_{o}$, which is subject to differentiability on a parameter $\mathrm{h}$, is defined by $r_{h}^{R_{o}}=\frac{\partial R_{o}}{\partial h} \times \frac{h}{R_{o}}$ [13].

Table 2. The following statistics were obtained. Iterations 10000, $\operatorname{Max} R_{0}=64.75307, \operatorname{Min} R_{o}=$ 55.87556 , Average $R_{0}=60.224$, Standard error in $R_{0}=1.438251$

\begin{tabular}{llllllllll}
\hline & Beta & Phi & delta2 & delta1 & Gamma & Tau & Theta & neta & mu \\
\hline Mean & 29 & 0.9 & 0.0519 & 0.0004 & 0.00001 & 0.006 & 0.200003 & 0.455 & 0.043082 \\
Standard & 0.5 & 0.05 & 0.0011 & 0.00005 & 0.000005 & 0.0005 & & 0.0005 & \\
Deviation & & & & & & & & & \\
\hline
\end{tabular}

Table 3. The following statistics were obtained. Iterations 10000, $\operatorname{Max} R=90.04521, \operatorname{Min} R=$ 65. 04974, Average $R=76.94214$, Standard error in $R=3.839519$

\begin{tabular}{llllllllll}
\hline & Beta & Phi & delta2 & delta1 & Gamma & Tau & Theta & neta & mu \\
\hline Mean & 29 & 0.9 & 0.0519 & 0.0004 & 0.00001 & 0.006 & 0.200003 & 0.455 & 0.043082 \\
Standard & 0.5 & 0.05 & 0.0011 & 0.00005 & 0.000005 & 0.0005 & & 0.0005 & \\
Deviation & & & & & & & & & \\
\hline
\end{tabular}

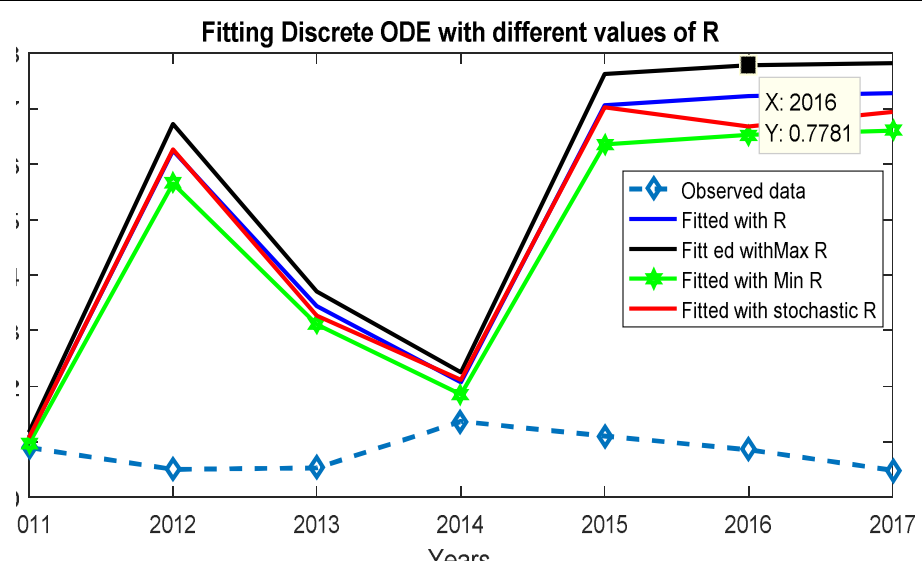

Fig. 11. Fitting data with different values of $\mathbf{R}$

Numerical results, which were obtained using the estimated parameters confirms that $E^{*}$ exist whenever $R_{0}>1$. $E^{*}$ was as $\{42,9.05\}$ and $\{24.8,75.8\}$ at $R_{0}=42$ and $R=22$ respectively 


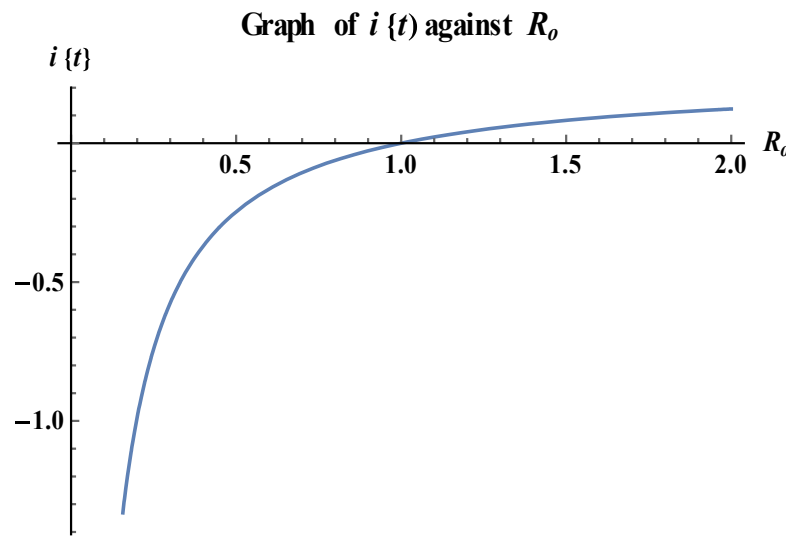

Fig. 12. Bifurcation analysis using $i(t)$

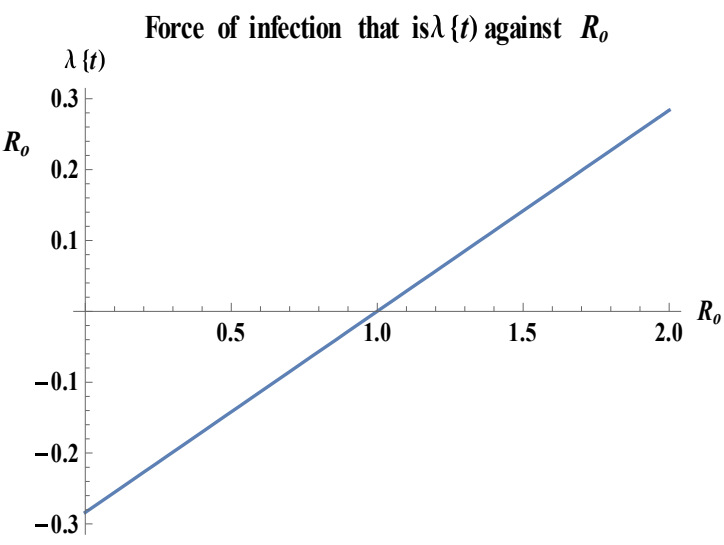

Fig. 13. Bifurcation analysis using $\lambda(t)$

Table 4. Normalized Sensitivity Analysis of Basic Reproduction Number

\begin{tabular}{ll}
\hline Parameters & Normalized sensitivity index \\
\hline $\mathrm{H}$ & 0.581267 \\
$\Phi$ & -0.201692 \\
$\theta$ & -0.568087 \\
$\mathrm{M}$ & -0.122368 \\
$\beta$ & 1 \\
$\partial_{1}$ & -0.000347982 \\
$\mathrm{~T}$ & -0.00521973 \\
$\mathrm{\Gamma}$ & $-1.97043 \mathrm{E}-05$ \\
$\partial_{2}$ & -0.102265 \\
\hline
\end{tabular}

Normalized sensitivity index on Basic Reproduction Number

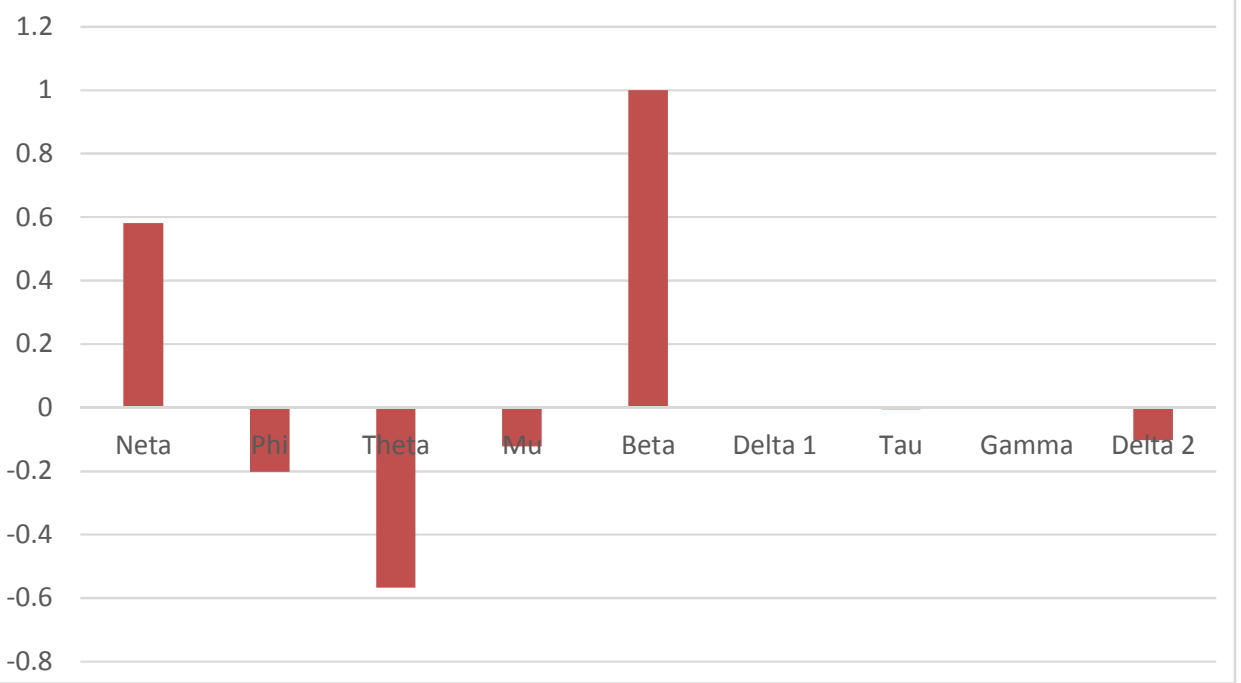

Fig. 14. Normalized sensitivity analysis 


\section{DISCUSSION}

The data obtained in Table 2 was not sufficient enough no wonder some estimation were inevitable. A case in point is where this had to use prevalence rate on study carried in neighboring country Ethiopia as a result of lack of such documented information in Kenya. In Fig. 2, the proportional infective children $(i(t))$ fitted to observed data with mean absolute error of 27.7923208 and standard deviation of absolute error of 25.1594. This cast doubt in the developed model and/or observed data of infective. Likewise, in Fig. 3, the proportional infective children $(i(t))$ abnormally fitted to observed data with mean absolute error of 43.19271 and standard deviation of absolute error of 46.11121 . This cast doubt in the developed model and/or observed data of treated children.

MATLAB simulations of dynamics of childhood pneumonia using estimated $R_{o}$ and $R$ in Figs. 4 to Fig. 7 indicates that $i(t)$ and $\mathrm{r}(\mathrm{t})$ attained Endemic equilibrium in 2014 and 2018 for $R_{o}$ and $R$ respectively. This concurs with pneumonia induced death in Kenya which seems to have stabilized from $15 \%$ to $17 \%[3,5]$.

Fitting of discrete ordinary differential equation to observed data using $\mathrm{R}$ instead of $R_{o}$ had great promise in reducing absolute error by 2.851533 . Despite good encouragement in use of R, Figs. 8 and 9 confirms observation made in Figs. 2 and 3.

Figs. 10 and 11 amplifies the impact of uncertainties in parameter to important thresholds in mathematical epidemiology like basic reproduction number. The results of iterations indicate that policy makers should consider $55.87556 \leq R \leq 90.04521$ when making decision. However, Fig. 12 suggests that we should consider minimum $\mathrm{R}$ in fitting discrete ordinary differential equations.

Figs. 13 and 14 show that the system exhibited no backward bifurcation which is a good gesture to pneumonia interventionist. This means that any effort which can reduce reproduction number to less or equal to one is sufficient to eradicate the menace. Reducing infection rates through contacts hold promise in eradicating pneumonia.

\section{CONCLUSION}

It is more effective and efficient to use effective reproduction number $(R)$ of 7 instead of basic reproduction number $\left(R_{o}\right)$ in mathematical modelling of Infectious diseases focusing on proportion of population because it's close to probabilistic basic reproduction number of 5.6 obtained by [19]. The findings cast doubt on model formulation and/or observed data. This study should give rise to new generation on pneumonia model and focus on collection of reliable and sufficient data.

\section{ACKNOWLEDGEMENTS}

This research did not receive any specific grant from funding agencies in the public, commercial, or not-for-profit sectors.

\section{COMPETING INTERESTS}

Authors have declared that no competing interests exist.

\section{REFERENCES}

1. Ngari CG, Malonza DM, Muthuri GG. A Model for Childhood pneumonia Dynamics; 2014.

2. WHO. Pneumonia-WHO. WHO; 2017.

3. Roux DM, Myer L, Nicol MP. Incidence and severity of childhood pneumonia in the first year of life in a South African birth cohort: the Drakenstein Child Health Study. Lancet Glob Health. 2015;95-103.

4. Pina JC, Alves de Moraes S, Martins de Freitas IC, Falleiros de Mello D. Role of Primary Health Care in child hospitalization due to pneumonia: a case-control study. Rev. Latino-Am. Enfermagem; 2017.

5. UNICEF. Key demographic indicators. UNICEF Data; 2017.

6. Jain MK, lyengar SRK, Jain RK. Numerical Methods for Scientific and Engineering Computation. New Delhi: New Age International Limited Publishers; 1984.

7. Herceg D, Dejana $H$. Arduino and Numerical Mathematics. In Proceedings of 9th Balkan Conference on Informatics (BCl'19). Sofia, Bulgaria: ACM; 2019.

8. Guoa L, Liub Y, Zhouc T. Data-driven polynomial chaos expansions: A weighted least-square approximation. Journal of Computational Physics; 2019.

9. Murray JD. Mathematical Biology I. An Introduction Third Edition. Newyork: Springer; 2000.

10. Savin I, Pushkarev A. Monte Carlo Methods in Economics. 2015;1-84. 
11. Wang ZX, Li DD, Zheng $\mathrm{HH}$. Model comparison of $\mathrm{GM}(1,1)$ and $\operatorname{DGM}(1,1)$ based on Monte-Carlo simulation. Physica; 2019.

12. Waykar SR. Mathematical Modelling: A way of life. International Journal of Scientific \& Engineering Research. 2013;4(4).

13. Brauer F, Driessche PVD, Wu J. Mathematical Epidemiology. Canada: Springer; 2008.

14. Li J, Blakeley D, Smith RJ. The Failure of Ro. Computational and Mahematical methods in Medicine; 2011.

15. Health MO. Kenya Health Information system. Nairobi: Ministry of health; 2017.
16. UNICEF. State of the world's children 2015 country statistical information. Gigigiri: UNICEF; 2015.

17. Bank W. World Development indicators. Google Public Data Explorer; 2018.

18. Fekadu GA, Terefe MW, Alemie GA. Prevalence of pneumonia among underfive children in Este town and the surrounding rural kebeles, Northwest Ethiopia; A community based cross sectional study. 2014;2(3):150-155. DOI: 10.11648/j.sjph.20140203.12

19. Otieno OfJ, Mugisha J, Oleche P. A probabilistic estimation of the basic reproduction number: A case of control strategy of pneumonia. Science Journal of Applied Mathematics and Statistics. 2014;53-59.

(c) 2020 Ngari and Kitavi; This is an Open Access article distributed under the terms of the Creative Commons Attribution License (http://creativecommons.org/licenses/by/4.0), which permits unrestricted use, distribution, and reproduction in any medium, provided the original work is properly cited.

Peer-review history:

The peer review history for this paper can be accessed here: http://www.sdiarticle4.com/review-history/58158 